CrossMark \& click for updates

Cite this: Nanoscale Horiz., 2016, 1,268

Received 16th November 2015 Accepted 3rd February 2016

DOI: $10.1039 / c 5 n h 00112 a$

rsc.li/nanoscale-horizons

\section{Redefining the functions of nanocapsule materials}

\author{
D. Crespy, ${ }^{\text {ab }}$ L. P. Lv and K. Landfester ${ }^{a}$
}

\begin{abstract}
Nanocapsules are key components in new technologies related to biomedicine and materials science. However, their long-term fate after use is still largely ignored. We discuss here a sustainable approach where the products of degradation of the nanoparticles play a significant role in their application because they are also functional molecules. The polymer shell of the nanocapsules is chemically engineered so that the degradation products formed upon chemical damage are useful after their normal use.
\end{abstract}

\section{Introduction}

Nanocapsules are used as dispersions or embedded in matrices for biomedical and materials applications. However, the longterm fate of the nanoparticles after their use is still largely ignored. Moreover, most of the materials in nanoparticles and nanocapsules are actually "dead materials" in the sense that their degradation products do not play an important role. These materials are either inert or even detrimental to their applications if their degradation products are toxic or lower the properties of the matrix in which they are embedded. One possibility to overcome this issue would be to recycle nanoparticles that do not degrade easily. Metallic nanoparticles are recovered by inducing liquid-liquid phase separation by heating microemulsions, ${ }^{1}$ decantation of inorganic nanoparticles used as catalysts and as Pickering stabilizers, ${ }^{2}$ or filtering nanoparticles using a supramolecular membrane. ${ }^{3}$ However, polymer nanoparticles are difficult to re-disperse and remain intact. Moreover, recycling nanoparticles is not a concern for biomedical applications.

We discuss here a sustainable approach where the products of degradation of nanoparticles play a predominant role because they are also functional molecules. This approach considers the lifetime of the nanoparticles. Therefore nanoparticles have to be carefully designed, keeping in mind that the material composing the nanoparticles is used to yield functional degradation products. The approach can be combined with retrosynthetic analysis ${ }^{4}$ with additional conditions concerning the degradation products. Similarly to the renewable carbon index (RCI) for green chemistry,

\footnotetext{
${ }^{a}$ Max Planck Institute for Polymer Research, Ackermannweg 10, 55118 Mainz, Germany. E-mail: crespy@mpip-mainz.mpg.de

${ }^{b}$ Vidyasirimedhi Institute of Science and Technology (VISTEC), 555 Moo 1 Payupnai, Wangchan, Rayong 21210, Thailand
}

we define here the active molecule index (AMI). The AMI is the percentage of active molecules within all degradation products of the nanoparticles. We briefly review here the different strategies to synthesize nanocontainers that release functional molecules upon their degradation and discuss their AMI values. We envision the future of colloid chemistry for which the Latin adage "multum in parvo" (much in little) will be truly applied by designing efficient nanocontainers that will serve not only during but also after their lifetime.

\section{From classical synthesis to the design of sustainable nanocontainers}

Classically, the synthetic molecules produced by industry are designed following retrosynthetic analysis taking into consideration the cost and availability of raw chemicals. Because of the new concerns of society regarding sustainability, several principles of synthesis were introduced as green chemistry labels. ${ }^{5,6}$ One important value linked with sustainable chemistry is the so-called renewable carbon index (RCI). The RCI is the percentage of carbon in the synthesized molecule that originates from a renewable feedstock. ${ }^{7}$ Typically, the carbon atoms from renewable feedstocks come from fatty acids or glycerol for example. ${ }^{8,9}$ Nowadays, further requirements imply that the renewable feedstock shall not compete with the food chain. This is the case for instance for L-lactide, cellulose, or terpenes. This means that the main concerns are that the raw material is cheap and is derived from renewable resources.

The function of nanocontainers can be typically fulfilled at low concentrations. ${ }^{10}$ This means that concerns about cost and renewable feedstock are less important because they do not represent a large fraction of the materials compared to the matrix in which they are embedded. The most important criteria to 
design the nanocontainers are their functions, the encapsulation efficiency, and their degradation, ${ }^{11}$ especially when they are employed for biomedical applications. However, nanotoxicology is an issue and there is no consensus yet on the harmonization of experimental procedures to investigate the toxicity of nanoparticles. ${ }^{12}$ For biomedical applications, the polymer matrix can be degraded by hydrolysis, oxidation, or enzymatic reactions. ${ }^{13}$ However, the nanoparticles can have a detrimental effect after their primary function is achieved. It was reported for instance that the degradation of nanoparticles of the popular poly(D,L-lactide), poly( $\varepsilon$-caprolactone), and poly(lactide-co-glycolide) displayed high toxicity for all the tested cell lines at high concentration. ${ }^{14}$ Usually, the amount of polymer matrix in nanoparticles for biomedicine is much larger than the amount of drugs that is encapsulated in the polymer matrix. Similarly, the polymer shells of microcapsules used for self-healing materials are usually non-useful materials. After the delivery of the healing agents, empty microcapsules remain in the matrix and become a preferential location for crack propagation or attack from external media. They are thus detrimental to the properties of the materials. We discuss here how the synthetic approach employed to design nanocontainers can be modified to implement a useful function to the nanocontainers even after their normal use.

\section{Chemical encoding of functional molecules in nanocontainers}

A classical method to lower the toxicity of the drug is to fabricate a prodrug that is later metabolized to yield the active form of the drug in the body. Nanocontainers are often used to encapsulate and protect prodrugs. For instance, nanoparticles based on chitosan derivatives were used as nanocarriers for neuropeptides to prevent plasma degradation. ${ }^{15}$

The chemical encoding of active substances in a macromolecular chain was introduced by Ringsdorf for the delivery of drugs $^{16}$ (see Fig. 1). The polymer comprised several segments that have different roles: a segment ensuring the solubilization of the polymer, the pharmacon to be delivered in the body, and one unit for targeted delivery. Many of such polymers do not contain the unit for targeting and are called polymer prodrugs. A now classical method to transport and release drugs is to conjugate them with a polymer. ${ }^{17,18}$ Such a strategy can be employed to formulate nanoparticles that are constituted

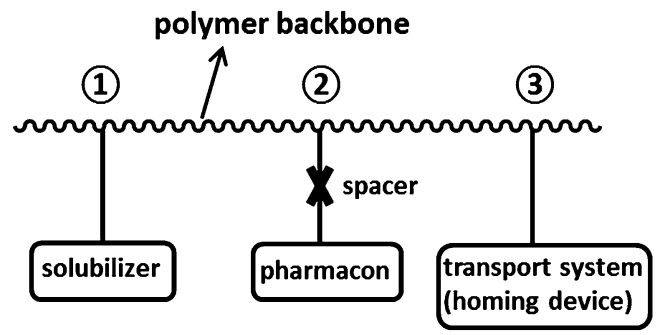

Fig. 1 Model of a pharmacologically active polymer. Adapted from ref. 16. of the polymer-drug conjugate. Several approaches were identified: the conjugation of a drug to a pre-synthesized polymer, the conjugation with a monomer followed by polymerization, and the initiation of a polymerization by a functionalized drug. ${ }^{19}$ The anticancer drug cisplatin was conjugated to a diblock copolymer forming micelles via hydrazone bonds and the drug could be released to the acidic medium of the tumor cell. ${ }^{20}$ The advantage of such an approach is that the drug is protected during its transport to the tumor cells. Gemcitabine, also an anticancer drug, was conjugated to reversible addition-fragmentation chain transfer (RAFT) agents for polymerizing squalenyl methacrylate ${ }^{21}$ and methyl methacrylate. ${ }^{22}$ The polymers could self-assemble to form nanoparticles in water that allowed for a pH-dependent release of gemcitabine. Similarly, alkoxyamines for nitroxide mediated polymerization (NMP) were functionalized with the same drug to yield nanoparticles. ${ }^{23}$

However, the quantity of drug compared to the polymer is still low. A method to increase the amount of useful active material incorporated, the so-called AMI value, is to use a drug or another active substance as a monomer or to graft it as a side chain of the polymer with a high grafting ratio.

The concept is particularly simple to achieve when polymers are synthesized by polyaddition or polycondensation reaction because the active molecules to be released can be directly used as monomers and be incorporated in the main chain of the polymer. This is the case for instance for $p$-coumaric acid ${ }^{24}$ or morphine $e^{25}$ that was used to form biodegradable poly(anhydride-ester) (Fig. 4a and b) with an AMI of 69 and 52\%, respectively. If the drug possesses only one reactive group for polycondensation as is the case with the anti-inflammatory drugs naxopren $(\mathrm{AMI}=62 \%)$ and ibuprofen (AMI $=64 \%$ ) that have one carboxylic acid functionality, it can be grafted as a pendant group in the side-chain of polyesters (Fig. 4c). ${ }^{26}$

The AMI number can also be significantly increased by grafting the drugs on the side-chains of polymers. Up to $43 \mathrm{wt} \%$ of paclitaxel could be introduced in the side-chain of the polyacrylic acid segment of a block copolymer. ${ }^{27}$ The polymers could self-assemble into nanoparticles in water and high antitumor activity was observed in vitro. Another method is to use the drug as a monomer in block copolymers that form nanoparticles in water. Thus, more than $50 \mathrm{wt} \%$ of the drug camptothecin could be loaded in nanoparticles by using it as a monomer prodrug that can be cleaved by reduction. ${ }^{28}$

Interestingly, these strategies can be hence converted for preparing polymer nanocontainers that can release active substances during their degradation as shown in Fig. 2. L-Arginine, which can be employed for dentin hypersensitivity, was copolymerized by interfacial polyaddition in water-in-oil miniemulsions to form nanocapsules. ${ }^{29}$ This means that the chemical encoding of active molecules can be further implemented into polymer nanocontainers. An example of a very efficient system was reported by Kwon et al. ${ }^{30}$ Nanoparticles were formed with the polymer poly(vanillin oxalate). The particles could deliver the anti-inflammatory and antioxidant vanillin molecule after oxidation and acidification. An elegant approach is to create a 


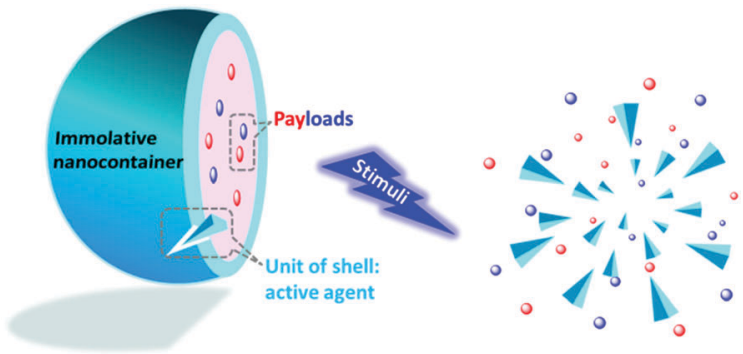

Fig. 2 Scheme of the nanocontainers with encoded active substances that are released during their degradation.

drug-drug conjugate to form nanoparticles. The hydrophilic irinotecan and the hydrophobic chlorambucil, both anticancer drugs, were conjugated via a hydrolysable ester linkage. ${ }^{31}$ The amphiphilic molecules were used to create nanoparticles, which displayed a longer blood retention half-time compared to free drugs. This allowed for the accumulation of the prodrug in tumor tissues and the ester bond could be cleaved by hydrolysis after cellular internalization. Anticancer activity was demonstrated in vitro and in vivo.

\section{Encoding the active agents for promoting the co-release of multiple payloads from nanocontainers}

The encoding of the active payload to be released in a polymer which forms the nanocontainers can be also used to release a second payload during the degradation of the nanocontainers. This approach was demonstrated by synthesizing amphiphilic copolymers with solubilizing units (polyvinylpyrrolidone) and units bearing the functional molecules to be released (Fig. 4d). ${ }^{32}$ The active payload was selected to be the corrosion inhibitor mercaptobenzothiazole that was bound with a disulfide linkage to the main chain of the copolymer. Nanoparticles were formed by self-assembly in water and were able to encapsulate an additional hydrophobic payload. The cleavage of the disulfide bonds led therefore to the simultaneous release of the corrosion inhibitor and the second payload. The AMI could be tuned between 2 and $22 \%$, depending on the ratio of monomers in the copolymer. Such an approach was also demonstrated in the case of large poly(anhydride-ester) microparticles based on salicylic acid. The anti-inflammatory drug salicylic acid could be released from the microparticles by hydrolysis, thereby triggering the release of encapsulated insulin. ${ }^{33}$ The AMI calculated by taking into account the produced salicylic acid and the released insulin was $70 \%$. In this case, the encapsulated insulin was distributed inside the monolithic polymer microparticles and not encapsulated in the core of a capsule.

The ratio of active molecules to be released can be substantially increased not only by employing the aforementioned strategy but also by designing the nanocontainers as core-shell nanoparticles with a functional liquid core instead of homogeneous monolithic nanoparticles. ${ }^{34}$ For this, block and random copolymers bearing corrosion inhibitors in the side-chain were used to form nanocapsules with a liquid self-healing agent physically entrapped in the core of the nanocapsules (Fig. 3). The release

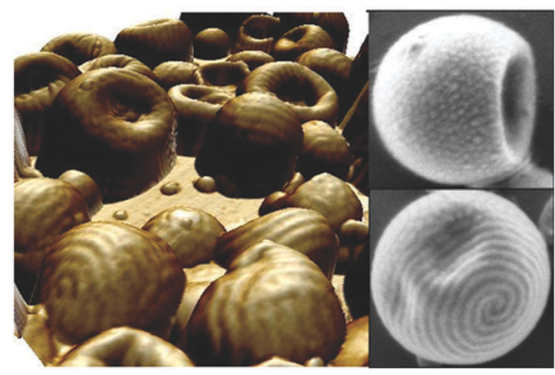

Fig. 3 AFM and SEM images of block copolymer nanocapsules with corrosion inhibitors chemically encoded in the shell for a triggered release of both the corrosion inhibitor and a liquid self-healing agent present in the core of the nanocontainers. ${ }^{34}$
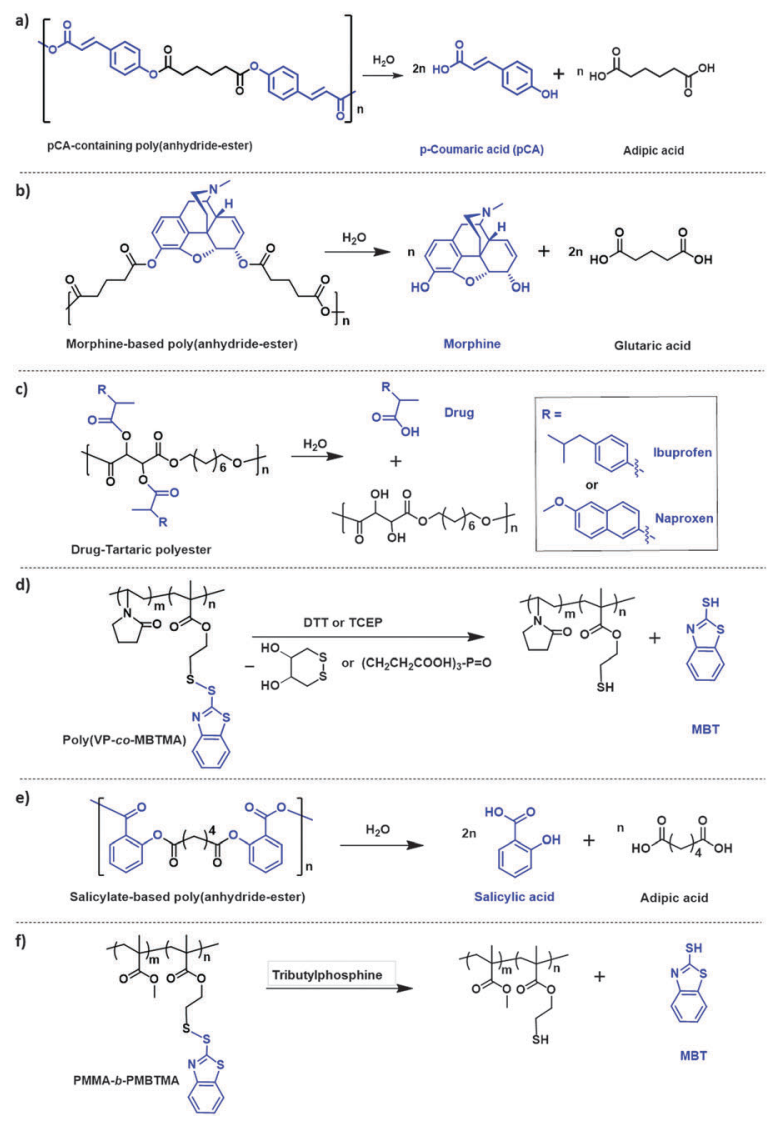

Fig. 4 Examples of copolymers with active molecules (marked in blue) as pendant groups in their main chains or side chains showing release of these active molecules after cleavage of the corresponding bond. ${ }^{24-26,32-34}$

of the corrosion inhibitors triggered a second release of the entrapped self-healing agent and the system could reach an AMI of $56 \%$. The release rate could also be controlled by the structure of the copolymer, i.e. random or block.

\section{Perspectives}

The systems proposed are still far from being fully developed and many interesting properties could be implemented in new 
nanocontainers to increase their efficiency and AMI value. The ideal nanocontainers would have an AMI value of $100 \%$ meaning that all materials forming the shell can be degraded to useful and active molecules (Fig. 2). The liquid core and the shell would therefore need to be degraded to form functional molecules. The functional molecules can be either chemically attached to the core or shell or physically bound. Such a concept is not only applicable to nanocontainers but also to other types of nanomaterials. The design could be combined with the self-immolative concept ${ }^{35}$ so that degradation could be accelerated upon demand. For biomedical applications, the concept could also be combined with photodynamic therapy, ${ }^{36,37}$ so that part of the degraded material becomes phototoxic while the rest of the degraded material is active but non-toxic. Indeed, toxicity issues in circulation and in vivo continue to be major issues that need to be carefully investigated before a product is approved for human trials. Finally, this approach could be employed to release very fragile or reactive ingredients as is the case in biology. For instance, preprohormones are produced in the body to protect hormones such as vasopressin that possess a very short lifetime. ${ }^{38}$

\section{Notes and references}

1 O. Myakonkaya, C. Guibert, J. Eastoe and I. Grillo, Langmuir, 2010, 26, 3794.

2 H. Liu, Z. Zhang, H. Yang, F. Cheng and Z. Du, ChemSusChem, 2014, 7, 1888.

3 E. Krig, H. Weissman, E. Shirman, E. Shimoni and B. Rybtchinski, Nat. Nanotechnol., 2011, 6, 141.

4 E. J. Corey, Angew. Chem., Int. Ed., 1991, 40, 455.

5 M. Poliakoff, J. M. Fitzpatrick, T. R. Farren and P. T. Anastas, Science, 2002, 297, 807.

6 J. Clark, R. Sheldon, C. Raston, M. Poliakkoff and W. Leitner, Green Chem., 2014, 16, 18.

7 M. McCoy, The Greening Game, Chem. Eng. News, 2009, 87, 13.

8 M. Toda, A. Takagaki, M. Okamura, J. N. Kondo, S. Hayashi, K. Domen and M. Hata, Nature, 2005, 438, 178.

9 U. Biermann, U. Bornscheuer, M. A. R. Meier, J. O. Metzger and H. J. Schafer, Angew. Chem., Int. Ed., 2011, 50, 3854.

10 G. Ibarz, L. Dahne, E. Donath and H. Mohwald, Adv. Mater., 2001, 13, 1324.

11 U. Edlund and A. C. Albertsson, Adv. Polym. Sci., 2002, 157, 67.

12 H. F. Krug, Angew. Chem., Int. Ed., 2014, 53, 12304.

13 E. Marin, M. I. Briceno and C. Caballero-George, Int. J. Nanomed., 2013, 8, 3071.
14 R. P. Singh and P. Ramarao, Toxicol. Sci., 2013, 136, 131.

15 A. Lalatsa, V. Lee, J. P. Malkinson, M. Zloh, A. G. Schatzlein and I. F. Uchegbu, Mol. Pharmaceutics, 2012, 9, 1665.

16 L. Gros, H. Ringsdorf and H. Schupp, Angew. Chem., Int. Ed., 1981, 20, 305.

17 R. Duncan, Nat. Rev. Cancer, 2006, 6, 688.

18 R. Haag and F. Kratz, Angew. Chem., Int. Ed., 2006, 45, 1198.

19 V. Delplace, P. Couvreur and J. Nicolas, Polym. Chem., 2014, 5, 1529.

20 S. Aryal, C. M. J. Hu and L. F. Zhang, ACS Nano, 2010, 4, 251.

21 D. T. Bui, A. Maksimenko, D. Desmaele, S. Harrisson, C. Vauthier, P. Couvreur and J. Nicolas, Biomacromolecules, 2013, 14, 2837.

22 W. Wang, C. Li, J. Zhang, A. Dong and D. Kong, J. Mater. Chem. B, 2014, 2, 1891.

23 S. Harrisson, J. Nicolas, A. Maksimenko, D. T. Bui, J. Mougin and P. Couvreur, Angew. Chem., Int. Ed., 2013, 52, 1678.

24 M. A. Ouimet, N. D. Stebbins and K. E. Uhrich, Macromol. Rapid Commun., 2013, 34, 1231.

25 R. Rosario-Meléndez, C. L. Harris, R. Delgado-Rivera, L. Yu and K. E. Uhrich, J. Controlled Release, 2012, 162, 538.

26 R. Rosario-Meléndez, W. L. Yu and K. E. Uhrich, Biomacromolecules, 2012, 14, 3542.

27 Y. Gu, Y. Zhong, F. Meng, R. Cheng, C. Deng and Z. Zhong, Biomacromolecules, 2013, 14, 2772.

28 X. Hu, J. Hu, J. Tian, Z. Ge, G. Zhang, K. Luo and S. Liu, J. Am. Chem. Soc., 2013, 135, 17617.

29 D. Crespy, M. Stark, C. Hoffmann-Richter, U. Ziener and K. Landfester, Macromolecules, 2007, 40, 3122.

30 J. Kwon, J. kim, S. Park, G. Khang, P. M. Kang and D. Lee, Biomacromolecules, 2013, 14, 1618.

31 P. Huang, D. Wang, Y. Su, W. Huang, Y. Zhou, D. Cui, X. Zhu and D. Yan, J. Am. Chem. Soc., 2014, 136, 11748.

32 L. P. Lv, Y. Zhao, K. Landfester and D. Crespy, Polym. Chem., 2015, 6, 5596.

33 R. Delgado-Rivera, R. Rosario-Meléndez, W. L. Yu and K. E. Uhrich, J. Biomed. Mater. Res., Part A, 2014, 102, 2736.

34 Y. Zhao, R. Berger, K. Landfester and D. Crespy, Small, 2015, 11, 2995.

35 A. P. Esser-Kahn, N. R. Sottos, S. R. White and J. S. Moore, J. Am. Chem. Soc., 2010, 132, 10266.

36 S. Y. Wang, G. Kim, Y. E. K. Lee, H. J. Hah, M. Ethirajan, R. K. Pandey and R. Kopelman, ACS Nano, 2012, 6, 6843.

37 N. M. Idris, M. K. Gnanasammandhan, J. Zhang, P. C. Ho, R. Mahendran and Y. Zhang, Nat. Med., 2012, 18, 1580.

38 Molecular Biology and Biotechnology: A Comprehensive Desk Reference, ed. R. A. Meyers, Wiley-VCH, 1995. 